\title{
Authors' reply: Expanding horizons in metastatic prostate cancer treatment
}

\section{Justin Sturge, Matthew P. Caley and Jonathan Waxman}

We thank Fizazi \& Goessl for their comments and interest in our article-Bone metastasis in prostate cancer: emerging therapeutic strategies. Nat. Rev. Clin. Oncol. 8, 357-368 (Expanding horizons in metastatic prostate cancer treatment. Nat. Rev. Clin. Oncol. doi:10.1038/nrclinonc.2011.67-c1 $)^{2}$-in which we focus on the current and emerging therapies for bone metastases in patients with castration-resistant prostate cancer (CRPC). We also thank them for bringing to our attention several errors in Tables 1 and 2 . These have been corrected for the HTML and PDF versions of the article. ${ }^{3}$

In our Review, we discussed the Medical Research Council (MRC) PR05 trial, which recruited men with prostate cancer and metastatic bone disease (MBD) who had started or responded to long-term hormone therapy. ${ }^{4}$ The long-term survival data from the final analysis included data from 278 patients $(89 \%)$ of the original 311 men recruited to the study, and 258 deaths (93\%) were reported. In the analysis by Dearnaley et al., ${ }^{4}$ a total of 31 patients (10\%) were excluded because they were not registered in England and Wales and a further two patients (1\%) were not identified by the UK National Health Service Information Centre. Evidence does not indicate that the $11 \%$ of patients excluded would have different response rates to clodronate. We remain in agreement with Dearnaley et al., ${ }^{4}$ who conclude a significant overall survival benefit from this first-generation bisphosphonate in patients with MBD. Further studies, for example the MRC-STAMPEDE trial, ${ }^{5}$ will ascertain if the more-potent bisphosphonate, zoledronic acid, is also of benefit to men starting long-term hormone therapy.

The superior efficacy of the fully human monoclonal antibody, denosumab, compared with zoledronic acid was given credence in our section titled: The new option: RANK-RANKL inhibition. ${ }^{1}$ The recent findings of the Amgen funded trial (NCT00104650) in men with CRPC and $\mathrm{MBD},{ }^{6}$ in which patients were randomly assigned to receive denosumab $(n=951)$ or zoledronic acid $(n=950)$, consolidates our proposal of this new option for the treatment of men with CRPC and MBD. The median time to first on-study skeletal-related event (SRE) reported was 20.7 months for denosumab compared with 17.1 months for zoledronic acid. The only notable difference in the frequency of adverse events was hypocalcemia, which occurred in $13 \%$ of patients treated with denosumab and $6 \%$ of patients treated with zoledronic acid. ${ }^{6}$ A second trial funded by Amgen (NCT00089674) 7 reported that denosumab improved bone mineral density and decreased vertebral fracture rate in patients with non-metastatic cancer receiving androgen deprivation therapy. These exciting findings provide an important adjunct to our review ${ }^{1}$ and give further encouragement to health-care providers for the successful treatment of patients with metastatic prostate cancer.
Division of Cancer, Department of Surgery and Cancer, Faculty of Medicine, Imperial College London, Hammersmith Hospital Campus, Du Cane Road, London W12 ONN, UK. (J. Sturge, M. P. Caley, J. Waxman)

Correspondence to: J. Sturge jsturge@imperial.ac.uk

Competing interests

The authors declare no competing interests.

1. Sturge, J., Caley, M. P. \& Waxman, J. Bone metastasis in prostate cancer: emerging therapeutic strategies. Nat. Rev. Clin. Oncol. 8, 357-368 (2011).

2. Fizazi, K. \& Goessl, C. Expanding horizons in metastatic prostate cancer treatment. Nat. Rev. Clin. Oncol. doi:10.1038/ nrclinonc.2011.67-c1.

3. Sturge, J., Caley, M. P. \& Waxman, J. Bone metastasis in prostate cancer: emerging therapeutic strategies [corrigendum]. Nat. Rev. Clin. Oncol. doi:10.1038/nrclinonc.2011.127.

4. Dearnaley, D. P., Mason, M. D., Parmar, M. K., Sanders, K. \& Sydes, M. R. Adjuvant therapy with oral sodium clodronate in locally advanced and metastatic prostate cancer: long-term overall survival results from the MRC PRO4 and PR05 randomised controlled trials. Lancet Oncol. 10, 872-876 (2009).

5. James, N. D. et al. STAMPEDE: Systemic therapy for advancing or metastatic prostate cancer-a multi-arm multi-stage randomised controlled trial. Clin. Oncol. (R. Coll. Radiol.) 20, 577-581 (2008).

6. Fizazi, K. et al. Denosumab versus zoledronic acid for treatment of bone metastases in men with castration-resistant prostate cancer: a randomised, double-blind study. Lancet $\mathbf{3 7 7}$, 813-822 (2011).

7. Smith, M. R. et al. Denosumab in men receiving androgen-deprivation therapy for prostate cancer. N. Eng. J. Med. 361, 745-755 (2009). 